\title{
8-YEAR-OLD BOY WITH PROGRESSIVE HEADACHE
}

\author{
Shawn L. Hervey-Jumper, MD'; Anthony C. Wang, MD'; Andrea N. Yates, BS ${ }^{1}$; \\ Patricia L. Robertson, MD²; Karin M. Muraszko, MD'; Hugh J.L. Garton, MD, MHSc; \\ Mila Blaivas, $\mathrm{MD}, \mathrm{PhD}^{3}$
}

Departments of ${ }^{1}$ Neurosurgery, ${ }^{2}$ Pediatrics, and ${ }^{3}$ Pathology, University of Michigan, Ann Arbor, Michigan, USA.

\section{CLINICAL HISTORY}

An 8-year-old boy with no significant past medical history presented to the emergency department following 2 weeks of progressive headaches, fatigue, and vomiting. On physical examination, the patient had no fever, or focal motor or sensory neurological deficits. Initially diagnosed with an H1N1 viral upper respiratory and gastrointestinal infection, he was discharged home with conservative management. After failure to improve over the next several days, a superimposed bacterial infection was suspected. He was given a course of oral antibiotics, which failed to improve his symptoms. Imaging revealed a large, heterogenously enhancing mass measuring $5.6 \times 4.7 \times 5.2 \mathrm{~cm}$, which contained partially hemorrhagic material centered within the left thalamus extending into the body of the lateral ventricle (Figures 1a). Gross total resection of the tumor was confirmed by postoperative imaging. Staging revealed no metastases. The patient was discharged home 1 week later.

\section{NEUROPATHOLOGICAL FINDINGS}

Grossly, the tumor submitted consisted of multiple pieces of friable, tan tissue fragments altogether measuring $2 \times 2 \times 0.5 \mathrm{~cm}$. Microscopically, the tumor was formed by pleomorphic, frequently multinucleated bizarre cells with numerous mitotic figures and multiple abnormal spindles (Figure 1b), multifocal tumor necrosis, calcifications, and exuberant microvascular proliferation (Figure 1c). Immunostaining of the sections showed many areas of neoplasm staining positively for synaptophysin (Figure 1d), and occasional cells positive for glial fibrillary acidic protein (GFAP) (Figure 1e) and vimentin. Positive Tuj1 staining was seen in some of these areas as well. Few cells were positive for S-100 protein, and none for NeuN or neurofilament protein. However, p53 as well as epidermal growth factor receptor (EGFR) (Figure 1f) showed robust multifocal staining. MIB-1 proliferation index was high, approaching close to $100 \%$ in some areas.

What is your diagnosis?
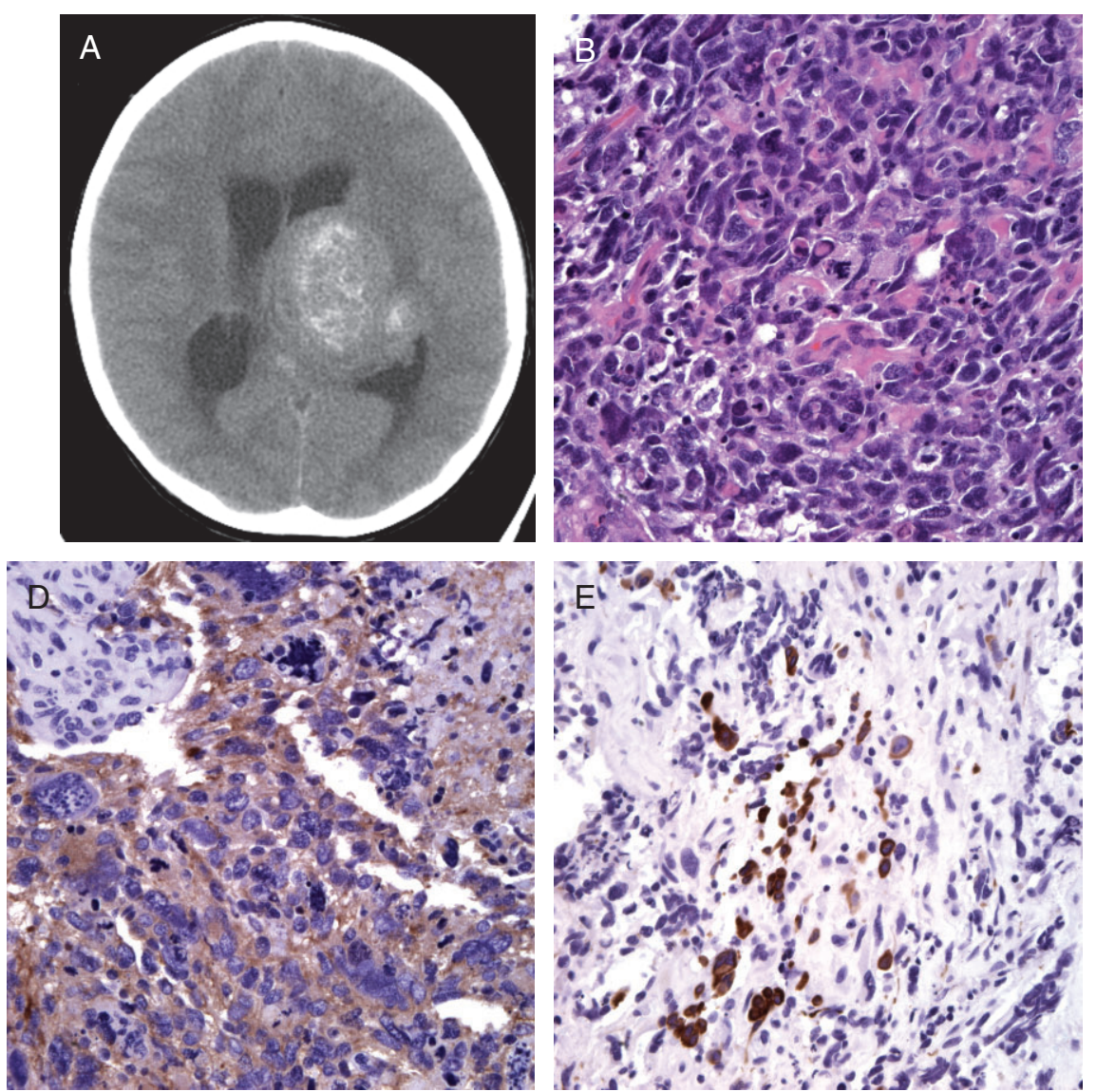
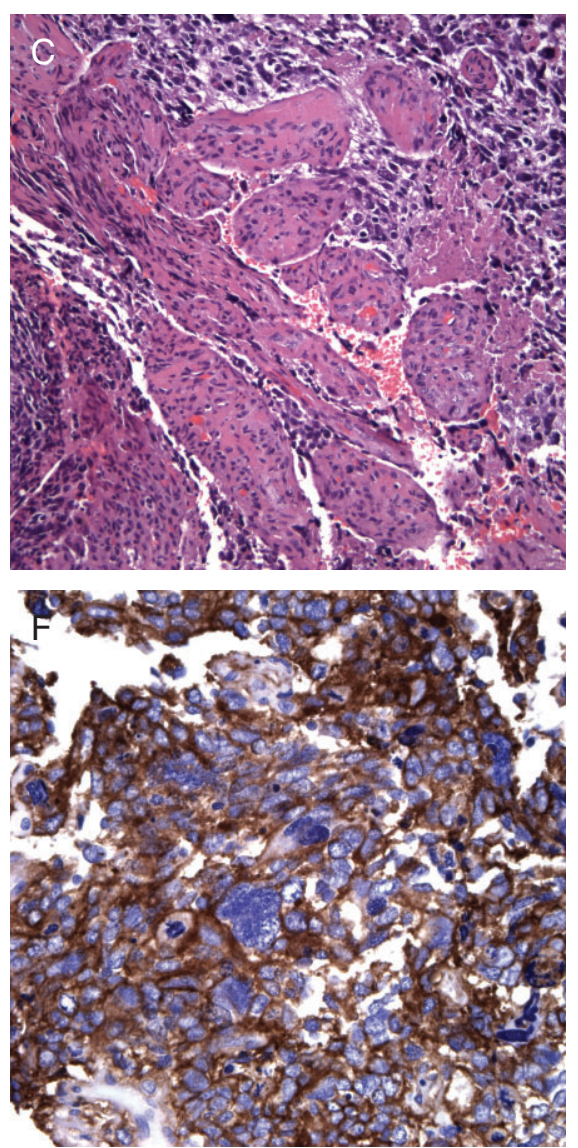

Figure 1. 


\section{DIAGNOSIS}

WHO Grade IV glioblastoma multiforme (GBM) with extensive neuronal differentiation.

\section{DISCUSSION}

GBM is the most common primary brain tumor occurring in the United States, accounting for an estimated 13,000 deaths each year (5). GBM is morphologically heterogenous with the histological hallmarks of extensive microvascular proliferation, prominent necrosis, and frequent mitoses. As an astrocytic neoplasm, GBM typically stains positively for GFAP and S-100 protein. In addition to the classic form of glioblastoma, 2 histological variants and several other patterns have been defined including gliosarcoma, giant cell GBM, glioblastoma with lipidized cells, small cell glioblastoma, glioblastoma with oligodendroglioma component, and glioblastoma with heterologous differentiation (1).

Gangliogliomas are typically well-differentiated neoplasms containing both neuronal and glial components. Though rare, several reports have described the malignant transformation of well-differentiated gangliogliomas into a GBM, with tumor microscopy revealing extensive astrocytic proliferation, high mitotic activity, endothelial proliferation, and cellular pleomorphism with multinucleated cells $(2,4)$. Both p53 mutations and inactivation of p16 have been implicated in the malignant transformation of gangliogliomas $(2,4)$.

No published reports have described a primary brain neoplasm exhibiting features of both glioblastoma as well as multiple regions of extensive neuronal differentiation. The documented experiences have been limited to case reports involving children and adolescents with malignant conversion of a known, well-differentiated ganglioglioma into GBM, as well as small case series of malignant glioneural tumors $(2,3)$. The diagnosis of malignant glioneural tumors is dependent on antibody positivity for neurofilament protein, NeuN, synaptophysin, and chromogranin, in addition to frequent mitoses (6). Such tumors pose unique questions regarding their biology and whether the two components reflect a "collision" between two separate neoplastic processes, or if the neuronal component arose from pluripotent precursor cells within the tumor.

In our patient, there was no indication of malignant conversion from ganglioglioma, and negative immunostaining for neurofilament protein and $\mathrm{NeuN}$ is inconsistent with the diagnosis of malignant glioneural tumor. The numerous areas of positive staining for synaptophysin or Tuj1 suggested extensive neuronal differentiation within the GBM. As such, following gross total resection, the patient was started on temozolomide followed by focal irradiation and erlotinib. He survived 257 days ( 8 months, 15 days) after diagnosis.

\section{ACKNOWLEDGMENTS}

The authors would like to thank Holly Wagner for providing editorial assistance in preparation of this manuscript.

\section{REFERENCES}

1. Figarella-Branger D, Bouvier C, Moroch J, Michalak S, Burel-Vandenbos F [Morphological classification of glioblastomas]. Neurochirurgie 56:459-463.

2. Hayashi Y, Iwato M, Hasegawa M, Tachibana O, von Deimling A, Yamashita J (2001) Malignant transformation of a gangliocytoma/ganglioglioma into a glioblastoma multiforme: a molecular genetic analysis. Case report. J Neurosurg 95:138-142.

3. Jeibmann A, Hasselblatt M, Pfister S, Strater R, Brentrup A, Holling $\mathrm{M}$ et al (2009) From glioblastoma to gangliocytoma: an unforeseen but welcome shift in biological behavior. $J$ Neurosurg Pediatr 4:475-478.

4. Kim NR, Wang KC, Bang JS, Choe G, Park Y, Kim SK et al (2003) Glioblastomatous transformation of ganglioglioma: case report with reference to molecular genetic and flow cytometric analysis. Pathol Int 53:874-882.

5. Louis DN, Ohgaki H, Wiestler OD, Cavenee WK, Burger PC, Jouvet A et al (2007) The 2007 WHO classification of tumours of the central nervous system. Acta Neuropathol 114:97-109.

6. Varlet P, Soni D, Miquel C, Roux FX, Meder JF, Chneiweiss H et al (2004) New variants of malignant glioneuronal tumors: a clinicopathological study of 40 cases. Neurosurgery 55:1377-1392. 